\title{
OPTICAL EQUIPMENT FOR MEASURING DEFORMATION OF MACHINE TOOL COMPONENTS
}

JAN KOUBEK, STEPAN CHLADEK, JIRI SVEDA, IVAN DIVIS

CTU in Prague, Research Center of Manufacturing Technology at Czech Technical University in Prague Prague, Czech Republic

DOI: 10.17973/MMSJ.2016_06_201612

e-mail: j.koubek@rcmt.cvut.cz

This paper describes the possibilities of measuring deformation of machine tool components based on monitoring change in the optical beam path. Deformation is evaluated relatively between an optical beam source and a sensing element. The great advantage lies in the fact that an active sensing element can be replaced by a passive optical element. No electronics needs to be installed in the measured place. Thus there is no necessity to protect electronic elements in a harsh environment. Another advantage is no need for statically arranged cables when measuring deformation on moving components. The measuring equipment presented in this paper consists of a measuring part and a part for measuring the motion of the reference beam. The position of a stiff body in space is clearly defined by 6 coordinates. The described optical equipment enables measurement of 4 coordinates, or 5 coordinates if an interferometer is used. The unmeasured coordinate describes the rotation of the measured body around the axis parallel with the optical beam.

KEYWORDS

Geometry, deformation, deviations, measuring, compensation, calibration, laser beam, horizontal boring machine

\section{INTRODUCTION}

Progress in most technologies in various industrial fields depends on technological capabilities of production machines. The priority of development is to improve the results of these technologies. If we want to maintain the universality of machines, we are (in most cases) limited by their design and physical properties of materials. In order to achieve better technological results, process monitoring needs to be improved. A process can be monitored from several perspectives of machine tool component behaviour. These together influence the resultant workpiece quality. Attention needs to be paid to adverse effects of machine too dynamic behaviour. These may occur when changing position in the machine tool workspace. Accelerating, braking and dynamic influence of technological forces may lead to adverse effects. It is especially the current technological condition of the machine [Weck 2006a] that determines dimensional and geometric accuracy of the machined part during stable machining. The condition of a machine is mainly influenced

Figure 1. Different structures of machines tools with marked critical parts.
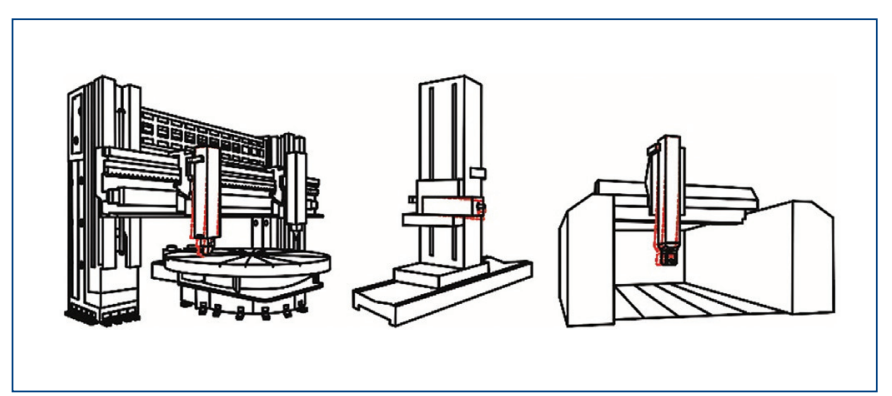

by the current state of machine adjustment, which is affected in particular by static, dynamic and thermal loading. Stiffness of components and their interfaces have a significant impact on changes in the geometry of the machine tool. Two types of changes can be distinguished. The first type of changes may be predicted after prior identification. The second type has the character of an accidental error, which cannot be described analytically [Tan 2005]. Identified phenomena can be compensated unlike random ones. In general, it applies that if these phenomena may be monitored during machining, it is possible to evaluate machine tool condition and to predict the resultant geometric shape of machined parts.

\subsection{Machine tools and Geometrical errors}

When considering larger-sized machines, it was discovered that, the maximum achieved accuracy changes according to different criteria than expected. The nature of an error, its direction and size may not be apparent at first. This is mainly caused by the wide spectrum of technological load, the type and arrangement of moving elements and the kinematic arrangement of a machine. In this paper attention will be given to larger-sized machines. These machines with a stroke in the order of units to tens of metres have a significant potential for increasing geometric accuracy and its long-term stability. Figure 1 shows a characteristic group of machines. Using a proposal of a specific system of measurement, the potential for improvement will be investigated.

Let us consider the structure of specific machines. Critical parts of machine tool structures show some common characteristics. Numerical methods are usually used for solving critical parts both in the design phase and in the phase of elementary investigation of the existing structure. Results achieved in this way may be verified by measurement in the case of a real structure [Benes 2011, Weck 2006b]. It follows that a common characteristic of machines of the above-mentioned type and size is dominant compliance in the ram. This is illustrated in [LIWEN 2012] using an example of a horizontal boring machine. This fact directly influences the real position of Theoretical Centre Point (TCP) in the machine tool workspace during machining. In the next part, the causes, possibilities and potential for enhancing this component will be discussed. The development of measuring equipment is motivated by the need to identify the current level of compliant parts deformation.

\subsection{Static deformation monitoring}

If the most compliant part from the machine tool structure is removed together with its load-bearing component (Figure 2), a relative change in the TCP position may be assumed. The position may change generally, in the sense of the six degrees of freedom, as indicated in the picture. The significance of particular changes (displacements and tilting) reflected in the resultant geometric workpiece errors varies. The conclusion may be reached that in very specific cases these particular changes do not affect the resultant geometric shape. A case in point is the tilting around the $Z$ axis, which does not adversely affect the geometric shape of the part in milling. This consideration presupposes co-linear arrangement of the cutting tool normal vector and the $Z$ axis in the sense of the main cutting motion. The influence of the change in TCP position on geometric errors of the workpiece is in detail explained in [Qianjian 2015].

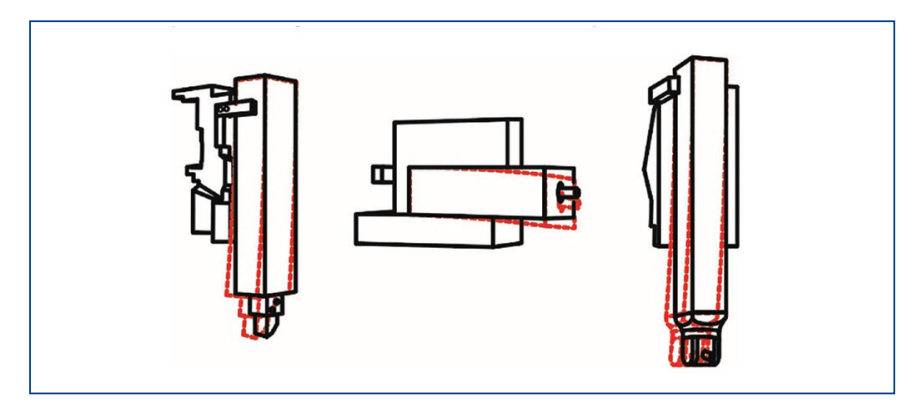

Figure 2. Details of critical parts of machine tools with indicated deformation. 
The monitored part of the machine consists of two basic components that usually have a common feed and continuous position control [Soucek 2004]. The mutual position is determined by the straightness of linear guideways, which take away five degrees of freedom to the extensible ram in the place of its interface, and the position feedback loop of the drive represented by one degree of freedom. Based on the above-mentioned facts, a general problem may be formulated with the following assumptions:

1) The cross slide is sufficiently stiff and its position in space can be defined.

2) The ram has significant static directional compliances,

which are represented by the displacement of TCP under static load.

The aim of our investigation is to search for a suitable method for monitoring the relative change in the position of the front part of the ram relative to the position of the cross slide. In order to develop the monitoring equipment, an idea was developed based on proposals of equipment for measuring deformation of the machine frames of CMM coordinate measuring machines [Huang 1995]. Corresponding design criteria were specified for the proposal of the system of measurement. Together with a proposal of a suitable concept of the optical path, possible arrangement and specifications were addressed in order for the equipment to be suitably installed together with appropriate electrical part in the structure of existing machine tools.

\section{DESIGN OF THE MEASURING DEVICE}

The optical equipment for measuring deformation of machine tool components consists of the optical beam source and a detector reading the point of the beam impact. The equipment uses propagation of light in a straight line in homogenous optical environment, when a change in the beam impact reflects a change in the optical equipment geometry or a change in the radiation angle of the beam source.

\subsection{Principle of the optical measurement}

The basic equipment consists of the optical beam source and a detector on which the optical beam falls. The evaluated change in the point of impact corresponds with a relative change in the geometry between the beam source and the detector. The above-mentioned configuration is shown in Figure 3a. This variant is the simplest configuration possible. It has several elementary drawbacks. The optical beam source and the detector of the point of impact are both optoelectronic devices that must be connected to a voltage supply. Therefore, it is necessary to install power-supply cables and signalling cables. This does not pose a problem if the configuration of the beam source and the detector is constant. However, if measurement of relative deformation between a moving source and detector is required, the positioning of power-supply cables may present a more difficult issue. Another limitation of the basic configuration of the measuring equipment is the limited selection of coordinates in which it is possible to monitor deformation. The limitation is defined by two mutually perpendicular displacements expressed on the beam detector plane.

The basic variant may be improved by placing a cube-corner reflector instead of an optoelectronic detector in the place of the measured deformation. As the reflector is a passive optical element, there is no need to install cables for measuring deformation on relatively moving parts. The beam source and the detector remain on a common platform. One of the properties of the cube-corner reflector is that the output optical beam is parallel with the input optical beam regardless of the tilting of the cube-corner reflector. Because of this it is not possible to measure deformation in rotation. Two mutually perpendicular displacements expressed on the plane of the beam detector may be measured. A great advantage compared to the original configuration lies in the fact that the cube-corner reflector displaced in a place perpendicular to the optical beam axis by a value of $\Delta$ reflects the input beam displaced by a value of $2 \Delta$. The main benefit of using this configuration is that the accuracy of deformation measurement is doubled.
It is not possible to use the above-mentioned options for measuring deformation in rotation. It is necessary to modify the optical equipment. The fundamental change is the replacement of the cube-corner reflector by an assembly of two mutually perpendicular plane mirrors, whose reflection surfaces form a right angle. The axis of this angle is parallel with the optical beam axis (Figure $3 \mathrm{~b}$ ). The assembly of two mirrors placed in this manner measures the displacement in one linear axis and simultaneously tilting around the same axis. It depends on the orientation of the mirrors in space in what axis the displacement and tilting will be measured. It applies as well that linear displacement of the mirror assembly by a value of $\Delta$ means a displacement of the input beam by a value of $2 \Delta$.

To measure two displacements in two mutually perpendicular axes and rotation around these two axes, further adjustment of the optical assembly needs to be done. One mirror is replaced by a one-way mirror so that the optical beam penetrates to the second mirror assembly, which measures displacement and rotation in the second axis (Figure 3c). Both beams are again reflected with double displacement relative to the displacement of the mirror assembly.

The change in the radiation angle of the beam source may have substantial influence on measurement accuracy and must be compensated in order to ensure sufficiently accurate measurement. Thus, it is necessary to monitor the point of the beam impact on the reference detector, which is placed in constant position to the beam source. It follows that the optical path length between the source and reference detector is constant (Figure 3d). A change in the point of beam impact on the reference detector is thus caused by a change in the radiation angle. To compensate this change in the detectors measuring displacement in particular directions, a thorough knowledge of the overall current geometry of measuring equipment is necessary. It is possible to determine the points of beam impact on particular optical elements and detectors based on the knowledge of the radiation angle and optical equipment geometry.

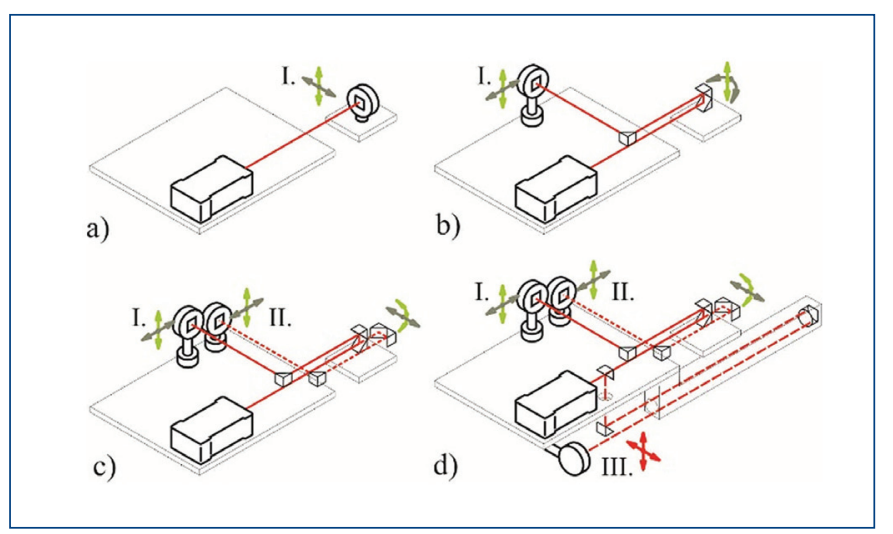

Figure 3. Different configurations of the optical measurement.

\subsection{Design of the measuring equipment adjusted to the ram}

The proposed design concept respects all requirements and possibilities of industrial application. The structure and materials used have to ensure appropriate measurement stability with respect to changes in the ambient environment. It is necessary to protect the space of the optical path between the basic part and the reflector by an appropriate cover, which has to protect sensitive measuring equipment from mechanical damage and to reduce thermal influence of the surrounding environment. Refraction index of air is dependent on temperature. If the optical beam passed through optical environment of various temperatures, the beam would be affected and measurement of accuracy would decrease due to a change in the refraction index. The design concept of the measuring equipment is shown in Figure 4. The equipment consists of two basic parts, a primary and secondary platform, which have constant configuration. The primary platform is composed of the laser beam 
source and Position Sensitive Device (PSD) sensors for measuring the change in the position of the secondary platform and divergence of the source itself. Mirrors and one-way mirrors are placed on the primary platform for optical path control. The primary platform is positioned in a place that serves as a reference point for measurement. The deformation measured by the secondary platform is determined by the position of the primary platform. The secondary platform consists of three mirrors and one one-way mirror. As these are only passive optical elements, there is no need to address the issue of power-supply cables and signalling cables. The secondary platform must be positioned on the moving body. The cube-corner reflector in the reference part ensures constant length of the optical beam path.

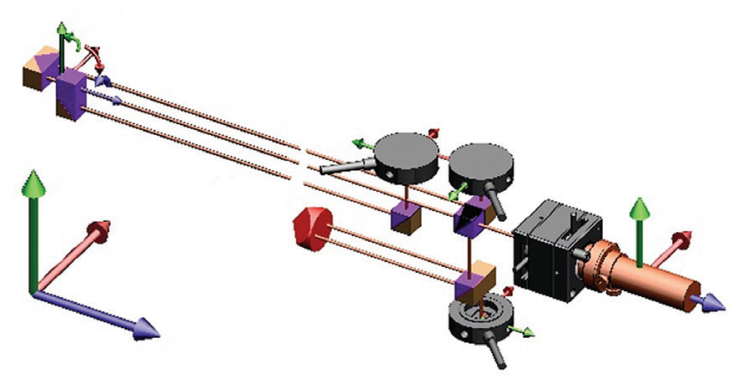

Figure 4. Design of the optical measurement device equipped with a reference part for beam divergence compensation.

\subsection{A mathematical model of the proposed equipment}

A toolbox was set up in the Matlab/Simulink software for the purposes of a mathematical description. This toolbox enables us to combine basic optical elements in an assembly and, based on their geometric arrangement, to calculate equations describing beam passage through the proposed equipment. The basic modelled elements include the optical beam source, mirror, one-way mirror, cube-corner reflector and PSD sensing element. The mathematical model of the beam source includes the input of output intensity and a description of output geometric properties. The mirror reflects the incident beam on the plane given by a normal vector of the reflection plane and a directional vector of the incident beam. Only the normal vector of the reflection plane is input. The one-way mirror divides the incident beam into two. The beam reflected from the reflection plane has the same properties as the beam reflected from the mirror and the passing beam with an identical directional vector of the input beam. The ratio of intensities of the passing and reflected beam are entered here as a parameter. The cube-corner reflector returns the output beam that is parallel with the input beam but has the opposite direction. The distance of the input and output beam depends on the point of input beam impact in the cube-corner reflector.

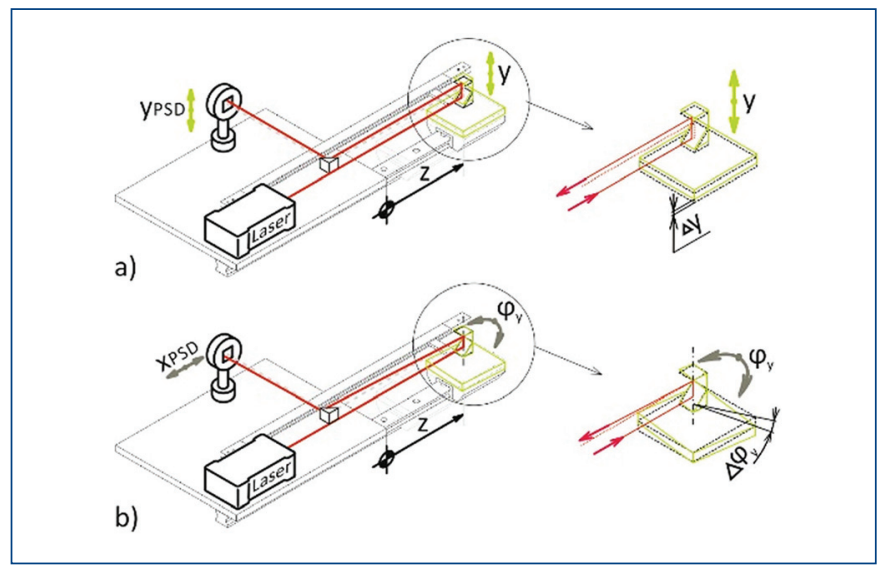

Figure 5. Scheme of the optical device for equation derivation.
The closer to the centre of the reflector the beam impacts, the smaller the distance of the beams are. The beam detector picks up the intensity and point of the input beam impact. Coordinates describing space arrangement of particular elements are input in the global coordinate system, which may be selected at random. Equations were derived by simulated displacement of individual components of the equipment together with subsequent linear regression of calculated values. The result is functional dependence expressing changes in the reflector coordinates and in the beam motion falling on the diode. The software allows graphical representation of the proposed optical equipment for a visual check of the geometric arrangement of the equipment and a possible check of inadmissible states. The aim is to derive equations describing dependence between the point of beam impact on sensing elements and measured displacements. For the optical equipment in the configuration expressed in Figure 5, allowing measuring displacement in the $y$ axis and rotation $\varphi_{y}$, the equations are derived in the form

$$
y=f_{1}\left(y_{P S D}\right), \varphi_{y}=f_{2}\left(z, x_{P S D}\right),
$$

where $x_{P S D}, y_{P S D}$ are the coordinates of the point of beam impact on sensing elements and $z$ is the variable distance in the measured configuration. Figure 5 does not show the geometric dimensions of the equipment. In order to derive equations (1), it is necessary to know the overall dimensional arrangement in the initial configuration.

\section{RESULTS AND DISCUSSION}

Testing equipment was designed for the purposes of model verification and real measuring equipment verification. The conditions for the testing equipment are the same as for a real installation. The extensible ram is mounted on rolling guideways. The testing apparatus is equipped with the linear ESSA measuring ruler for measurement of ram extension. The cover of the optical path is made from a transparent material. The design and the real measuring equipment is shown in Figure 6. The general arrangement of the testing equipment is shown in Figure 7. A device was selected for the experimental part consisting of an industrial PC equipped with the dSpace signal processor and controlled by the Matlab/Simulink software. Newport OBP-U-9Ha were selected as PSD sensing elements.

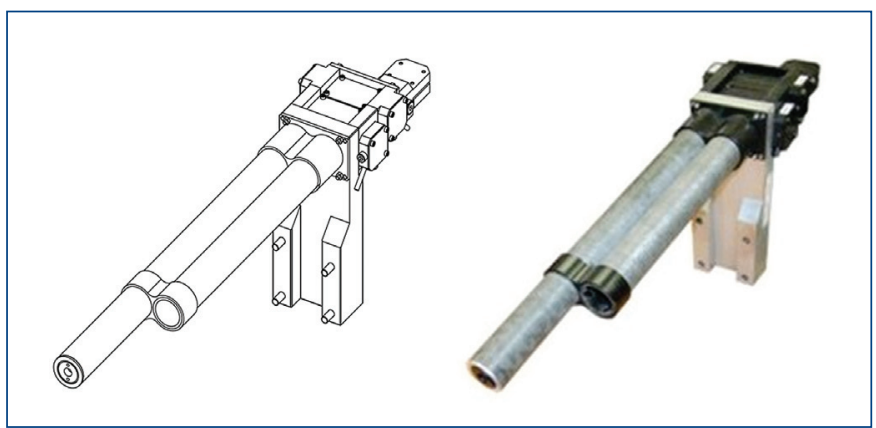

Figure 6. Primary platform of the optical equipment for deformation measurement. Design concept (left) and real installation (right).

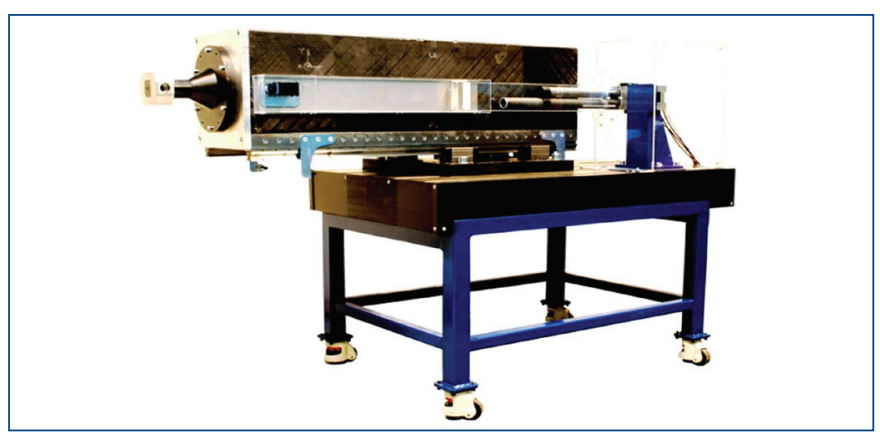

Figure 7. Test bed for model verification. 
The Thorlabs S1FC635 diode laser was selected as the optical beam source. The laser generates light beam with a wavelength of $635 \mathrm{~nm}$ and a maximum power of $2.5 \mathrm{~mW}$. The output power of the source may be regulated continuously. The equations (2-5) have the following form for specific arrangement of the optical equipment shown in Figure 7:

$$
\begin{gathered}
\varphi_{x}=\operatorname{arctg}\left(\frac{x_{D 2}}{-2 l+60.1}\right) \\
\varphi_{y}=\operatorname{arctg}\left(\frac{x_{D 1}}{2 l-163,7}\right) \\
x=-\frac{y_{D 2}}{2} \\
y=\frac{y_{D 1}}{-2}+22,5 \cdot \tan \left(\frac{x_{D 2}}{-2 l+60.08414}\right)
\end{gathered}
$$

The $x$ and $y$ values represent secondary reflector displacements; $\varphi_{x}$ and $\varphi_{y}$ represent tilting around indexed axes. The variable I corresponds to the ram displacement calculated for the distance between the beam source and PSD elements. Values measured on two measuring PSD diodes are represented by the variables $x_{D 1}, y_{D 1}, x_{D 2}, y_{D 2}$.

\subsection{Stability tests}

First, beam stability was measured as a time function on the test bed. The configuration of the measuring equipment remained constant for the whole time of measurement. Therefore, the change in the point of beam impact is caused by a change in the radiation angle. Graphs in Figure 8 show the characteristic of the points of beam impact on the reference sensing element. The four-quadrant Thorlabs PDQ80A diode was used instead of a PSD element to achieve higher accuracy. The diode produces higher accuracy of measurement. However, its measuring range is significantly smaller. The optical beam source was placed approximately $1.800 \mathrm{~mm}$ away from the four-quadrant diode. The experiment was prepared in such a manner that the beam in the initial position fell on the centre of the four-quadrant diode, i.e. to the coordinates $x=0, y=0$. The data were captured by frequency $f=0.5$ $\mathrm{Hz}$. The recording time was 72 hours. It is evident from the measured data that the point of beam impact changes in time by up to $160 \mu \mathrm{m}$. The biggest change occurs in the initial phase, in which the laser source heats up to operating temperature. Beam divergence in the range of $100 \mu \mathrm{m}$ is also visible after the initial phase.

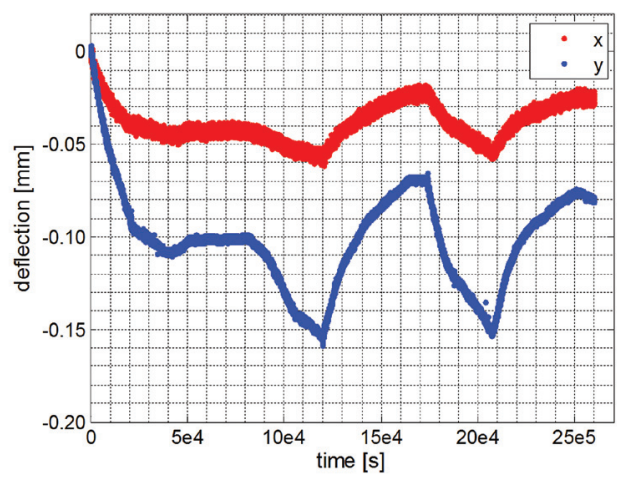

Figure 8. Divergence of the laser beam as a function of time measured on the reference diode. Red line represents the $x$ coordinate, blue line the $y$ coordinate.

\subsection{Beam divergence compensation}

Laser beam divergence was proved by measurement shown in 3.1 and has to be compensated to ensure sufficient accuracy. Using adjustable software, it is possible to derive equations describing beam transfer between the reference and measuring PSD elements. However,

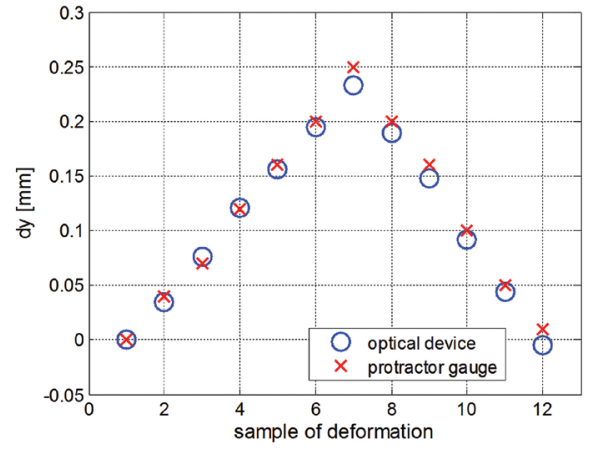

Figure 9. Deformation of the end of the test ram in the direction of the y axis.

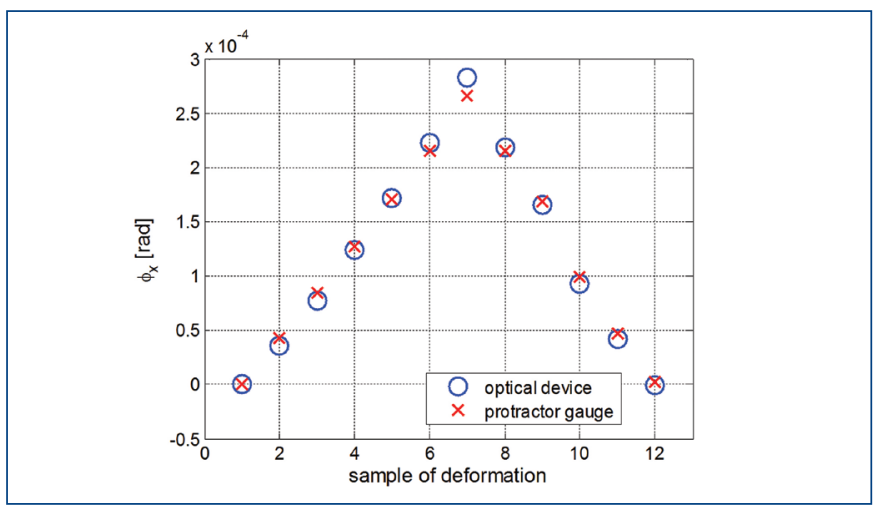

Figure 10. Angle deformation of the end of the test ram in the $\phi x$ direction.

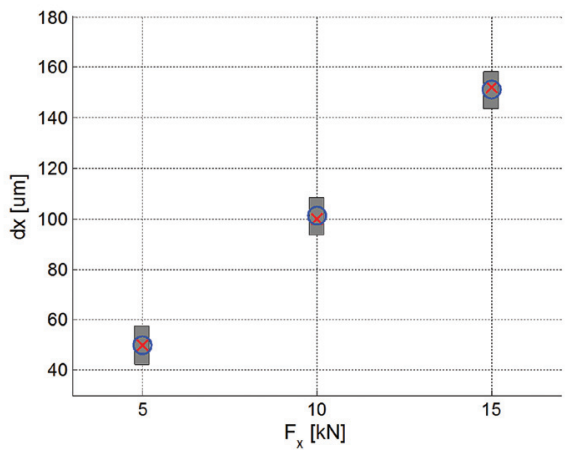

Figure 11. Deformation of real machine tool ram measured with dial indicator (red crosses) and the optical device (blue circles) in the direction of the $x$ axis.

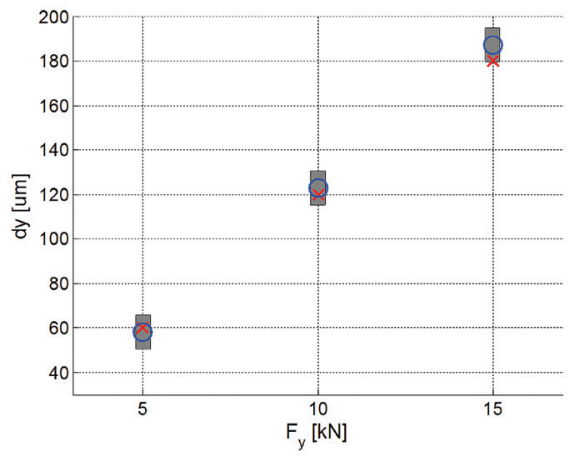

Figure 12. Deformation of real machine tool ram measured with dial indicator (red crosses) and the optical device (blue circles) in the direction of the y axis. 
these equations in their basic form do not include non-linearity and inaccuracies of the real structure. For this reason, it is more accurate to adjust the transfer using an experiment. The proposed procedure comprises displacing the beam source at a constant configuration of the measuring equipment and recording points of impact on reference and measuring sensing elements. Relative coordinates are calculated from the points of impact. These coordinates describe the mutual position between the measuring and reference sensing element. The relative position of two coordinate systems is expressed by their tilting, offset and distance from the optical beam source in the sense of the optical path of the beam. Based on relative coordinates, it is possible to compensate PSD measuring elements by deducing the value corresponding to beam divergence from the currently measured value. This value is then input into equations (2-5) to calculate deformation. The Newport 8821 displacing table was used for laser beam displacement. The table enables rotation around two mutually perpendicular axes. The motion of individual axes is ensured by two piezomotors allowing automation of the calibration measurement.

\subsection{Real deformation monitoring}

Real deformation monitoring is motivated by the need to know current geometry depending on load. As mentioned above, the measuring equipment does not measure in absolute values, but only in values relative to the selected reference value. The reference value for the testing equipment is determined by the stationary table top (Figure 7). Carriages of linear guideways of the extensible ram are attached to this table top. The displacement value is measured using the ESSA linear ruler. The end of the ram, approximately in the place of deformation measurement, was loaded with a force in the $y$ axis direction. For comparison, the deformation in y axis direction was measured with a dial indicator and the angle deformation was measured with a protractor gauge. The evaluated deformation values in the y direction is shown in graph in Figure 9 and torsion deformation $\varphi_{x}$ in graph in Figure 10. The resultant size of deformation corresponds to the stiffness of the test bed loaded with small forces.

Next, a measurement was performed, in which the optical equipment was mounted on a real machine tool ram and subsequently loaded with forces $F_{x}=\{5,10,15\} k N, F_{y}=\{5,10,15\} k N$ [KOUBEK 2011]. The calculated deformations were compared with deformations measured with a dial indicator. The data from the experiment are shown in graphs in Figure 11 and Figure 12. The accuracy of the PSD sensing elements used is up to $\pm 25 \mu \mathrm{m}$ according to the producer. The accuracy measured under laboratory conditions was $\pm 15 \mu \mathrm{m}$. The resulting insensitivity range of a given deformation is drawn with grey rectangles in the surroundings of the deformation value measured by the optical equipment. The insensitivity range of PSD elements in all cases included the value measured by a dial indicator. In most measured values, the value obtained using a dial indicator was in the centre of the insensitivity range.

\section{CONCLUSION}

The presented optical equipment for measuring deformation of machine tool components has several significant advantages over commercially available devices [Sveda 2011]. The first advantage is the passive secondary platform, which can be placed on a moving body without the need to address the arrangement and motion of cables. The motion of the secondary platform was successfully tested on the extensible ram. The second advantage is the compensation part of the equipment for measurement and correction of laser beam divergence. The divergence was measured experimentally. Although the divergence produces slow changes, which can be neglected in short-term measurements, it is necessary to compensate beam divergence in long-term measurements. Otherwise the resultant data contain many errors. Thirdly, one great advantage is the given arrangement of the optical path, where double values of the deformation in the direction of linear axes are measured in this arrangement. Thus, twofold accuracy is achieved compared to commercially available devices.

\section{ACKNOWLEDGEMENT}

The paper has received funding from the Technology Agency of the Czech Republic (Project TE01020075).

\section{REFERENCES}

[Benes 2011] Benes, P., Sveda, J., Divis, I. and Koubek, J. Testing Results of Mechatronic Technology. Prague: Research Center for Manufacturing Technology, 2011. Research Report. 23 p.

[Huang 1995] Huang, P. S. and Ni, J. On-line error compensation of coordinate measuring machines. In: International Journal of Machine Tools and Manufacture. 1995, pp. 725-738. ISSN: 0890-6955

[Koubek 2011] Koubek, J. Zusatzmessung für die Identifikation einer Geometrieveränderung und die Verformung einer Werkzeugmachine. Prague: CTU in Prague, FME, 2011, pp. 1-10. ISBN: 978-80-0104796-5.

[Liwen 2012] Liwen, G., Chunxia, Z., Huifeng, Z. and Huayang, X. Modeling and Analysis of a Super Heavy Numeric Control Boring and Milling Machine. IERI Procedia, 2012, pp.199-204. doi:10.1016/i. ieri.2012.06.031.

[Qianjian 2015] Qianjian, G. et al. Application of information fusion to volumetric error modeling of CNC machine tools. In: The International Journal of Advanced Manufacturing Technology. Springer, 2015, pp. 439-447. ISSN: 0268-3768

[Soucek 2004] Soucek, P.: Servomechanism in the machine tools, CTU in Prague, Prague 2004, ISBN 80-01-02902-6. (in Czech)

[Sveda 2011] Sveda, J., Divis, I. and Koubek, J. The Device for Optical Measurement of Accuracy and/or Deformation of Machine Tool Functional Sample. 2011.

[Tan 2005] Tan, K. K. A., Huang, S. Geometrical error compensation of machines with significant random errors. ISA Transactions, 2005, pp. 43-53. ISSN: 0019-0578

[Weck 2006a] Weck, M. A., Brecher, C. Werkzeugmaschinen, Messtechnische Untersuchung und Beurteilung, dynamische Stabilität. 7. neu bearb. Aufl.. Berlin: Springer, 2006. ISBN 3-540-22505-6.

[Weck 2006b] Weck, M. and Brecher, C. Werkzeugmaschinen, Konstruktion und Berechnung. 8. neu bearb. Aufl. Berlin: Springer, 2006. ISBN 3-540-22502-1.

\section{CONTACTS}

Ing. Jan Koubek

CTU in Prague, Faculty of Mechanical Engineering

Department of Production Machines and Equipment, RCMT

Horska 3, 12800 Prague 2, Czech Republic

P+420 221990 949, j.koubek@rcmt.cvut.cz,www.rcmt.cvut.cz

Ing. Stepan Chladek

CTU in Prague, Faculty of Mechanical Engineering

Department of Production Machines and Equipment, RCMT

Horska 3, 12800 Prague 2, Czech Republic

P+420 221990 957, s.chladek@rcmt.cvut.cz,www.rcmt.cvut.cz

Ing. Jiri Sveda PhD.

CTU in Prague, Faculty of Mechanical Engineering

Department of Production Machines and Equipment, RCMT

Horska 3, 12800 Prague 2, Czech Republic

P+420 221990 932, j.sveda@rcmt.cvut.cz, www.rcmt.cvut.cz

Ing. Ivan Divis

CTU in Prague, Faculty of Mechanical Engineering

Department of Production Machines and Equipment, RCMT

Horska 3, 12800 Prague 2, Czech Republic

P+420 221990 941, i.divis@rcmt.cvut.cz,www.rcmt.cvut.cz 